\title{
Reaction of arterial endothelial cells to stent impression: In vitro study using a model of the human artery wall
}

\author{
R.P. Franke ${ }^{\mathrm{a}}$, R. Fuhrmann ${ }^{\mathrm{a}}$, A. Krüger ${ }^{\mathrm{b}}$ and F. Jung ${ }^{\mathrm{b}, *}$ \\ ${ }^{a}$ Abteilung Biomaterialien, Zentralinstitut für Biomedizinische Technik, Universität Ulm, \\ Ulm, Germany \\ ${ }^{\mathrm{b}}$ Institute of Biomaterial Science and Berlin-Brandenburg Center for Regenerative Therapies, \\ Helmholtz-Zentrum Geesthacht, Teltow, Germany
}

\begin{abstract}
.
Stenoses in arterial blood vessels by artherosclerotic processes can decrease the supply of downstream tissue dramatically. The implantation of stents by percutaneous coronary intervention is one method of choice to restore the physiological blood flow. In some cases in-stent re-stenoses by thrombotic events, vascular wall hyperplasia or endothelial dysfunction occur. Causes and nature underlying this processes are not fully understood.

Aim of the present study was to study the re-stenotic processes after stent impress on a cellular and molecular level in vitro. Therefore, human arterial endothelial cells (HUAEC) were seeded on a model vascular wall intima consisting of extracellular matrix secreted by bovine corneal endothelial cells. Subsequently, a pre-mounted balloon-expendable tubular stent made of 316 L was impressed through the HUAEC layer leading to an impairment of the vessel wall intima. After stent removal the wound healing process, HUAEC membrane integrity, vitality, proliferation and function were assessed.

Immediately after stent impress an increased level of lactate dehydrogenase (LDH) was observed indicating an impairment of the cell membrane integrity. After $24 \mathrm{~h}$ baseline LDH values presented again. HUAEC vitality adjacent to the stent impress induced wound was normal (investigated by inverted microscopy). The proliferation of HUAEC was the highest in the direct vicinity of the stent impress induced wound. Prostacyclin and nitric oxide decreased significantly indicating a temporary loss of cell function.

These results could imply that the healing process of the endothelial cell lesion is superior to the maintenance of vascular tonicity and downregulation of platelet aggregation.
\end{abstract}

Keywords: Endothelial cells, stent impression, in vitro study

\section{Introduction}

Cardiovascular diseases are still the leading cause of death worldwide. The majority of fatal events are related to coronary artery disease, where the development of stenoses within the arteries narrows the arterial lumen depriving the supplied tissue of oxygen and nutrients. This disease cannot be cured using current therapies. One method to combat such stenoses or occlusions includes percutaneous transluminal

${ }^{*}$ Corresponding author: Prof. Dr. F. Jung, Institute of Biomaterial Science and Berlin-Brandenburg Center for Regenerative Therapies, Helmholtz-Zentrum Geesthacht, Teltow, Germany. E-mail: friedrich.jung@ hzg.de. 
coronary angioplasty (PTCA) with the implantation of a stent, which helps to prevent the arteries from becoming narrowed or blocked again.

During stent implantation the endothelial cells (EC) and their subcellular matrix are damaged or destroyed. The high pressure implantation is chosen to assure that the stenotic vascular region supplied with a stent has the same luminal diameter as those diameters found in the more or less healthy vascular regions before and after the stenotic region. This allows a sufficient blood supply again for the downstream myocardial tissues. Also the perturbation of and vortex generation in the blood stream entering and leaving the stented region might be avoided [4], which are thought to trigger an atherosclerotic development [6].

Ethical arguments do not allow a thorough in vivo examination of the causes and steps in the development of vascular re-occlusion after stent application. It is possible, however, to analyse these processes down to the cellular and molecular level in a vascular model in vitro.

In a plane model of a human blood vessel wall [8] the pathological development in EC was examined after impression of a stent into the model blood vessel wall followed by the subsequent removal of the stent from the experimental set up.

The vascular endothelium plays a key role in the development of the re-stenosis process in the stented vascular region. Endothelial dysfunction and/or hyperplasia of the vascular wall can induce a critical reduction of the vessel lumen $[12,13]$.

That is why in a first step the interaction between clinically applied stents and human arterial EC derived from healthy vessels and cultured under optimized conditions was examined. Based on earlier experience, the in vitro examination of human arterial EC from healthy vessels, which were untreated and not intoxicated is thought to allow an estimation of the vascular wall reaction on the implantation of a stent in a more or less healthy or dysfunctional vessel.

\section{Material and methods}

For the examination of the interaction of the vascular wall intima with stents on a cellular and molecular level, the stents were pressed in vitro in a model vascular wall intima consisting of EC derived from human arterial vessels cultured on a basal membrane - like extra cellular matrix [8]. The contact-induced trauma was assessed by micromorphological examination, through parameters indicative of cell damage, through secreted cell products and correlated to regional rates of EC proliferation [20, 32].

\subsection{Stents}

Nine pre-mounted balloon-expendable tubular coronary stents (type "Multilink"TM", Abbott Vascular, Illinois, USA, Fig. 1) were used, which are in interventional cardiology in practise since years [5, 17, $30,34]$.

\subsection{Study design}

\subsubsection{Endothelial cells}

EC of the third passage derived from primary EC from human umbilical cord arteries (HUAEC) were incubated in standard culture medium supplemented with human serum pool (HSP $[15,16])$. Every second day the culture medium was exchanged. 


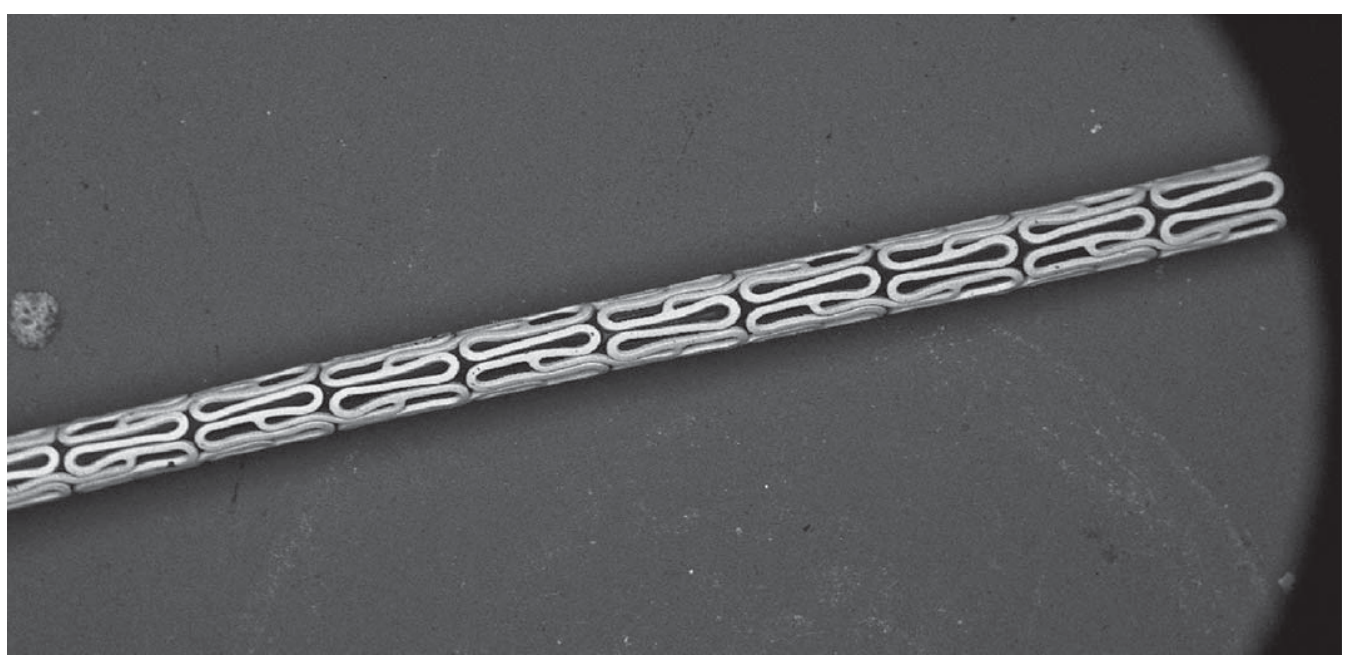

Fig. 1. SEM Image of a MultiLink ${ }^{\mathrm{TM}}$ stent prior to dilation (primary magnification 1:9).

The HUAEC were grown in vitro on an extracellular matrix (on glas slides with a diameter of $2.7 \mathrm{~cm}$ ) secreted by bovine corneal ECs and typical of medium and large vessel intimae and maintained until functional confluence (stress fibre reduction and formation of a marginal filament band) under static standard culture conditions $\left(5 \% \mathrm{CO}_{2}, 95 \%\right.$ humidity, $\left.37^{\circ} \mathrm{C}\right)$ [8]. HUAEC were experimentally exposed to stents 28 days after onset of culture.

\subsubsection{Test procedure}

With an apparatus designed for this purpose [9] the stents were pressed in the model intima in a laminar flow hood. During impression the stents penetrated the endothelial layer and the sub-endothelial basal membrane-like extra cellular matrix. To study wound healing in the stent-induced endothelial lesions but without the presence of the stents (the stents were removed from the culture vessels immediately after stent impression). Wound healing was intermittently analysed, micro-photographs were taken and evaluated. The impression apparatus allowed to test stents in a plane vessel model and to apply an initial pressure between stent and the HUAEC cultures similar to the implantation pressure in blood vessels (see Fig. 2). First, the stent mounted on a balloon catheter was placed on a functionally confluent HUAEC culture situated in a specially designed chamber filled with culture medium. Then, a second HUAEC culture, mounted upside down on a hydraulic cylinder, was placed on the stent immersed in culture medium from above. The pressure in the hydraulic cylinder was adjusted to 9 bar.

Functionally confluent HUAEC of the same passage and after the same period of time in culture which were not exposed to stents were used as controls.

To assess the level of damage done to the HUAEC by the stent impression, the amounts of nitric oxide (NO), prostacyclin and of lactate dehydrogenase, an indicator of the loss of barrier function of cell membranes, were evaluated from the cell culture medium.

On day 28 in culture the culture medium was removed, centrifuged, then fast frozen down to $-18^{\circ} \mathrm{C}$ and stored until measurement. These data were used to assess the quality and maturity of the cell cultures. Culture media were replaced by fresh culture media and left for two hours. Then samples of culture media were drawn, centrifuged and frozen to measure later on the "pre-stent-impression" values of prostacyclin, 


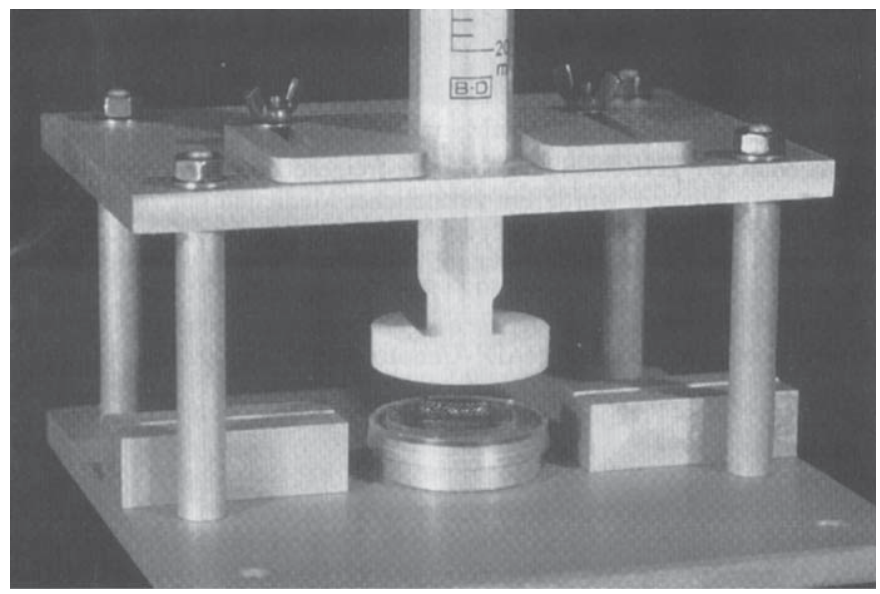

Fig. 2. Device for the impression of the stent into the planar vessel wall using a dilation balloon catheter.
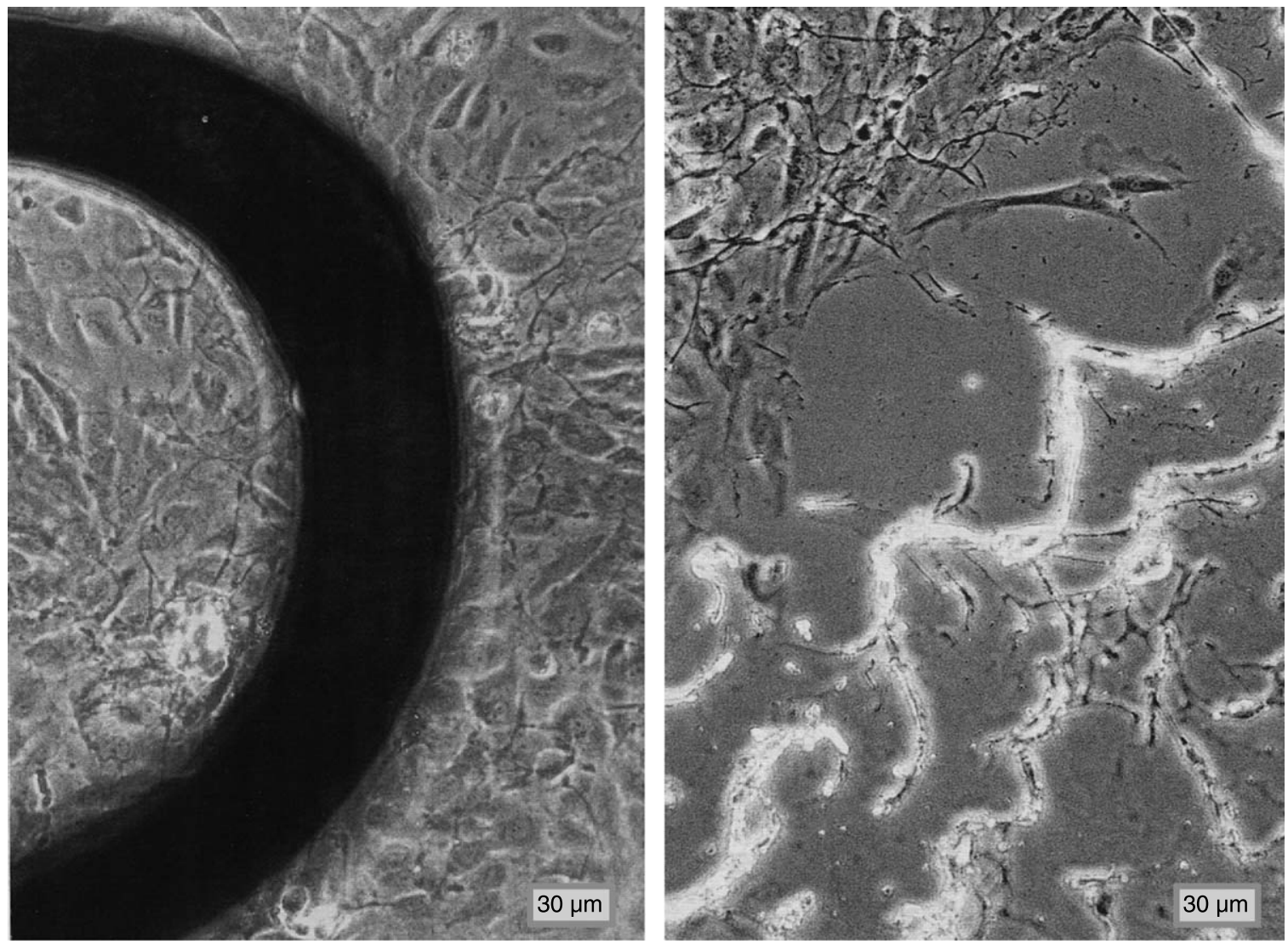

Fig. 3. Model vascular wall consisting of functionally confluent human arterial endothelial cells (HUAEC) on extra cellular matrix (ECM) with impressed stent (left image; primary magnification: 1:200) and after removal of the stent (right image, primary magnification: 1:200). 
$\mathrm{NO}$ and LDH. The removed small culture medium volume used for measurement was isovolumetrically replaced by fresh culture medium. Immediately thereafter, the stents were impressed in the cell cultures ( 9 bar inflation pressure) for 30 seconds and then removed. Again, samples of culture media were drawn, centrifuged and frozen to measure later on the "post-stent-impression" values of prostacyclin, NO and $\mathrm{LDH}$. The removed small culture medium volume was also replaced by fresh culture medium and culturing was continued for 24 hours after stent impression. Then, again, samples of culture media were drawn, centrifuged and frozen to measure later on the 24 hours values of prostacyclin, $\mathrm{NO}$ and LDH. The HUAEC cultures were aldehyde fixated and processed for further analyses.

In addition, the endothelial layers were examined with respect to cell proliferation and cell vitality. Cell vitality was assessed from cell morphology.

\subsection{Methods}

Prostacyclin was measured with the EIA-assay (Cayman Chemicals, Ann Arbor, Michigan, USA) according to instructions of the manufacturer [24]. NO-release was measured by evaluation of the stabile endproducts exploring the Griess reagent [27]. As an indicator of cell membrane damage, lactate dehydrogenase (LDH) was quantified exploiting the enzymatical metabolization of $\mathrm{NAD}^{+}$to NADH [18].

HUAEC proliferation was evaluated applying the S-phase marker bromodeoxyuridine (BrdU) [14]. 23.5 hours after the impression of stents in the arterial wall model BrdU was added to the HUAEC

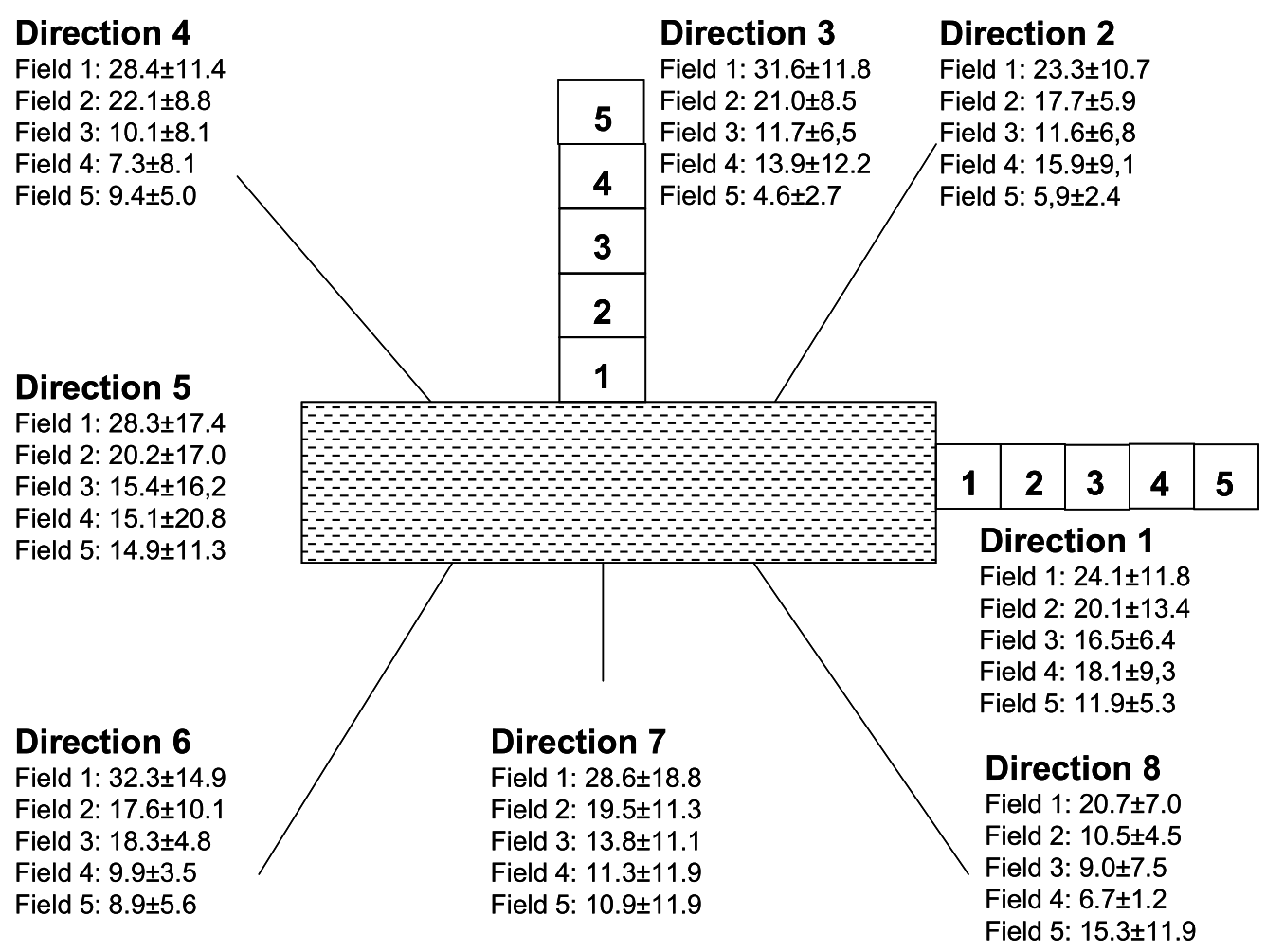

Fig. 4. Numbers of proliferating HUAEC over all of the 8 radial directions and specified for the 5 view fields. 
cultures and left there for 30 minutes. Then the culture medium was withdrawn and the cells were aldehyde fixated.

Since the growth factor FGF $(10 \mathrm{ng} / \mathrm{ml})$ was added for cultures of HUAEC, the growth rate was increased. The proliferation rates induced by stent impression were evaluated in radial directions along 8 pre-selected linear routes (see Fig. 4). Starting from the wound border and moving along the selected routes away from the wound area all the HUAEC per field of view which took up BrdU were counted. 5 fields of view were evaluated per selected route with the first field contacting the wound border followed by the fields 2 to 5 in radial direction to the outside.

The HUAEC cultures were examined in an inverted microscope on every exchange of culture medium. 24 hours after stent impression micro-photographs were taken of the state of the cells in the vascular wall model.

\subsection{Statistics}

For all samples mean values and standard deviation are given. Two sample comparisons were performed (prostacyclin, NO) using $t$-Test, or in case of non-Gaussian distribution Wilcoxon-Mann-Whithney UTest. For three sample comparisons ANOVA for repeated measures was utilized. $P$-values less than 0.05 were considered significant.

\section{Results}

\subsection{Assessment of the function of endothelial cells}

24 hours after stent impression the amounts of prostacyclin measured considerably decreased in relation to the starting values. There was a $62.6 \pm 4.99 \%$ decrease from starting value $(15.8 \pm 1.25[\mathrm{ng} / \mathrm{ml}])$ to the post impression value $(5.9 \pm 0.72[\mathrm{ng} / \mathrm{ml}])$. The difference in the mean values of the two groups was greater than would be expected by chance; there was a statistically significant difference between the input groups (two-sided $t$-test: $p<0.001$ ).

NO-values also decreased considerably 24 hours after stent impression. Here a $72.5 \pm 25.6 \%$ decrease was found from starting values $(0.051 \pm 0.009[\mathrm{mM}])$ to the post impression values $(0.014 \pm 0.012$ $[\mathrm{mM}])$. The difference in the median values between the two groups was greater than would be expected by chance; there was a statistically significant difference (Wilcoxon Mann Whitney U-test: $p<0.001$ ).

After the stent impression into the vascular wall model, the evaluation of the released amount of LDH in relation to the LDI amount before stent impression was performed. Directly after stent impression, LDH values were much higher $(0.43 \pm 0.06[\mathrm{mU} / \mathrm{ml}])$ than before stent impression $(0.13 \pm 0.03[\mathrm{mU} / \mathrm{ml}])$. The increase amounted to $231 \pm 37.8 \%$. 24 hours after additional cell culture medium exchange the LDH values returned to $0.16 \pm 0.24[\mathrm{mU} / \mathrm{ml}]$, which was near to the starting values.

\subsection{Assessment of cell morphology and vitality}

Following the impression of the $n=9$ stents in the vascular wall model, between $1.3 \%$ and $2.6 \%$ of the HUAEC in the vicinity of the impressed stents were completely destroyed. The subendothelial extra cellular matrix (ECM) was well to recognize (Fig. 3).

The cell vitality was assessed from cell morphology. 24 hours after stent impression all HUAEC, with the exemption of those cells destroyed by the stent impression, regardless whether they were close to or 


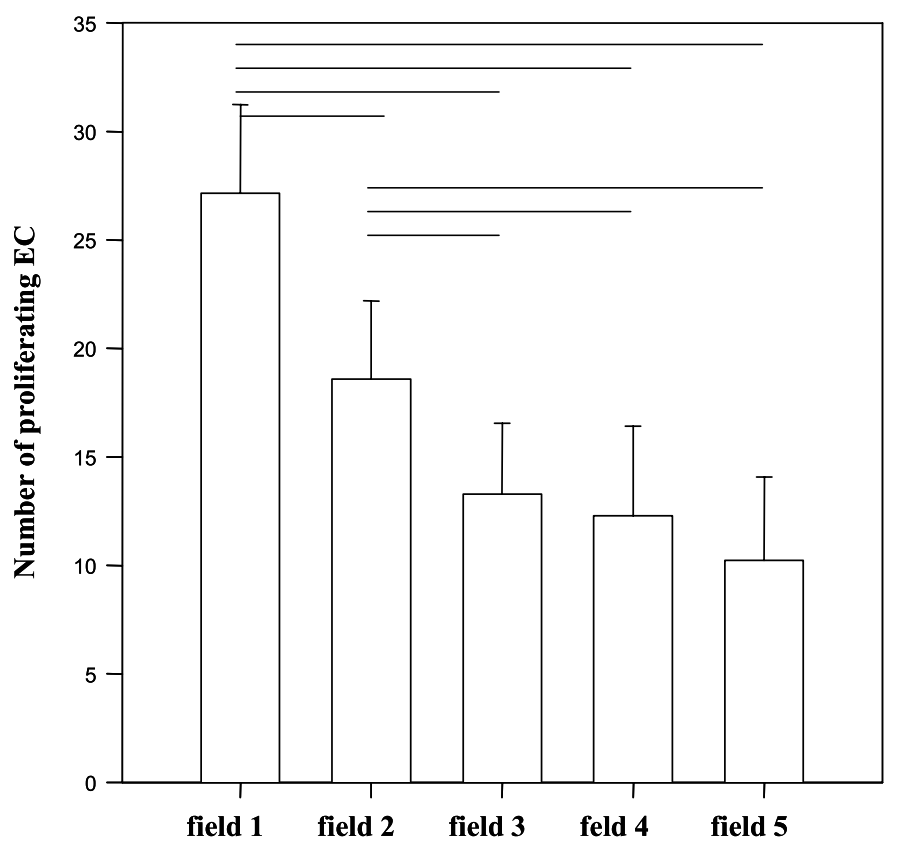

Fig. 5. Averaged numbers of proliferating HUAEC in the five fields (bars indicate $p<0.05$; Holm-Sidak multiple comparisons test).

remote from the area of damage were in good shape and vital. This was found around all of the impression related areas of damage. There was no difference to the vitality of HUAEC in control cultures. There was no difference to the vitality of HUAEC in control cultures.

In a birds view the stents and their corresponding impression induced wound areas appeared rectangular where the enter-/exit-sides were smaller than the long sides. Figure 5 schematically shows the localisations of the documented view fields around the wound areas and the average numbers of proliferating HUAEC per view field.

Maximum values of BrdU incorporation and proliferation rates appeared in view field "1" directly at the border of the wound areas and decreased significantly in radial directions from field " 1 " to field " 5 " (ANOVA: $p<0.001$ ).

Figure 5 shows the numbers of proliferating HUAEC averaged over all the 8 radial directions, but specified for the 5 view fields.

While the numbers of proliferating HUAEC directly at the long sides of the border (view fields 1; see Fig. 6) of the stent impression-induced wound area did not differ from the numbers of proliferating HUAEC directly at the entry-/exit-sides of the wound border (view fields 5; see Fig. 6), there was a significant difference and asymmetry in the view fields, which were farthest remote from the wound border. Here (in view fields 5), the numbers of proliferating HUAEC at the entry-/exit-sides were clearly greater than at the long sides of the stents $(t$-test: $p<0.023)$.

\section{Discussion}

Endothelial lactate dehydrogenase (LDH) is a stabile enzyme leaving the endothelial cytosol and gaining access to the extra cellular space only after disruption of the endothelial cell membrane integrity 


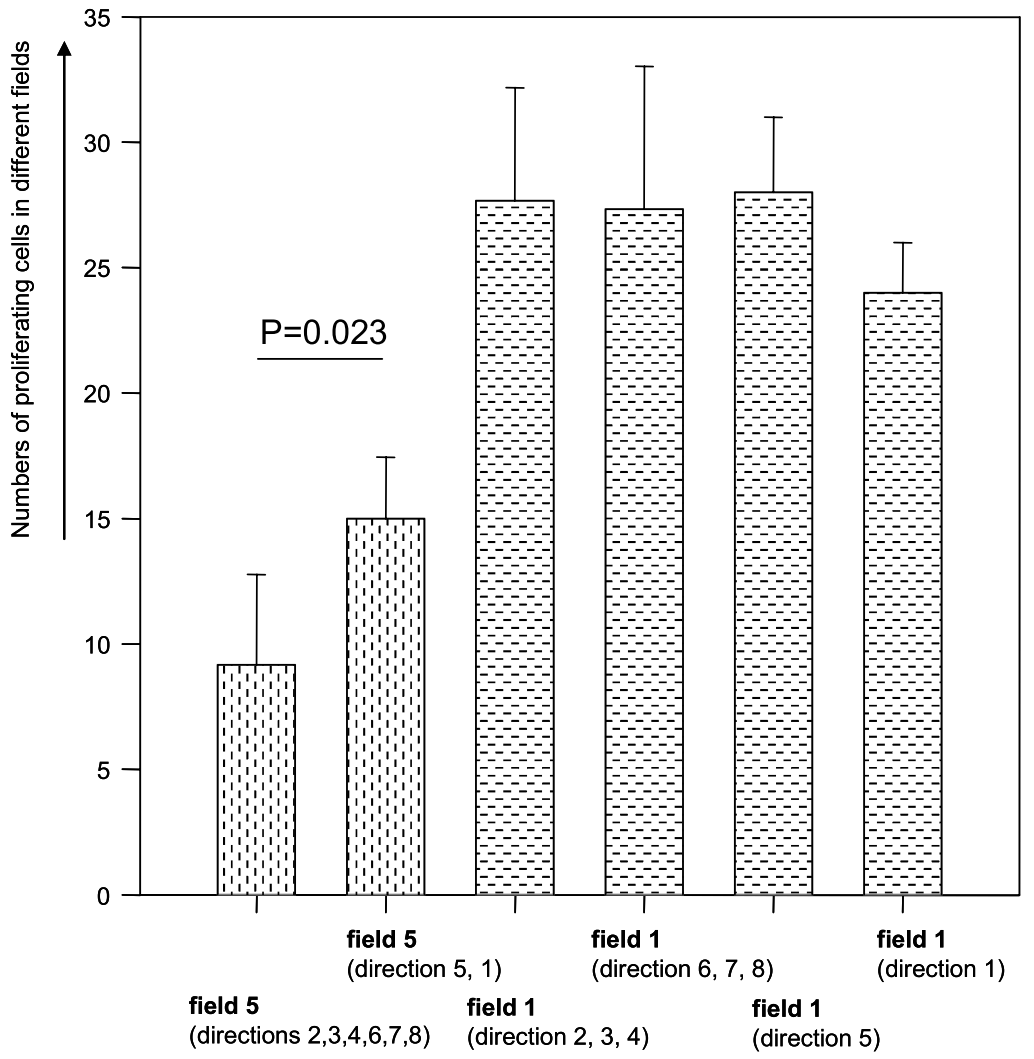

Fig. 6. Numbers of proliferating HUAEC in fields directly at the stent (view fields 1) and in fields which were farthest away from the stent border (view fields 5).

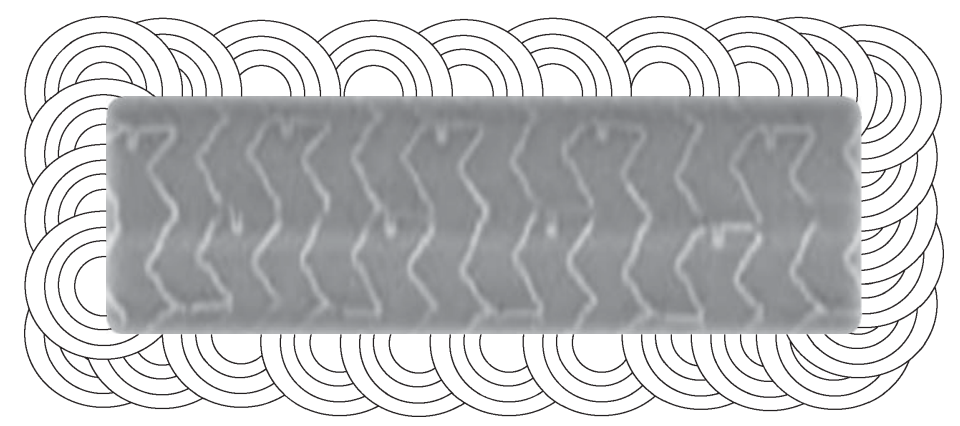

Fig. 7. Pattern of the diffusion of growth factors from the stent surface around the wound after stent impress.

or after complete damage of endothelial cells $[10,18]$. LDH is therefore well suited to assess the extent of cell damage of endothelial cells directly after stent impression and also later on. In comparison to base line values directly before stent impression there was a significant increase in free LDH of $231 \pm 37.8 \%$ immediately after stent impression. At this point the culture medium was completely removed, used to measure the LDH values and replaced by fresh culture medium. This happened in order to simulate the 
physiological situation where all the impression wound-related metabolites with access to the passing blood stream would have been carried away. None of the substances/factors released into the blood by stent impression-caused HUAEC destruction would be locally available. $24 \mathrm{~h}$ later the values of free LDH had returned to the baseline level. That is a clear hint that no self-perpetuating process culminating in cell destruction was initialised. The situation was different with the parameters used for the characterisation of endothelial function.

The values of prostacyclin as well as of nitric oxide (NO) were clearly below baseline values $24 \mathrm{~h}$ after stent impression. Prostacyclin plays an important part in avoiding platelet aggregation, in the regulation of the vascular tonicity and in the endothelial cytoprotection [25, 33]. The secretion of prostacyclin from endothelial cells is an important indicator of endothelial performance capacity. Following the impression of stents in the HUAEC monolayer there was a considerable decrease in prostacyclin secretion. And $24 \mathrm{~h}$ after stent impression there was still an important average decrease in prostacyclin secretion of $61.5 \pm 5.2 \%$. This decrease was much bigger than the decrease in the numbers of destroyed HUAEC $(1.3 \%-2.6 \%)$. Also the increase of proliferating HUAEC in the border zone of the stent impressioninduced wound areas, coinciding with a minimum of prostacyclin secretion from proliferating HUAEC, could not explain the significant decrease in prostacyclin secretion. The significant and hyper-proportional decrease is thought to be caused by a decrease in prostacyclin synthesis. A release of e.g. thromboxane, tumour necrosis factor or thrombin from perishing endothelial cells was described which could activate endothelial cells $[11,31,35]$ and possibly deteriorate the functional confluence and lead to a temporary endothelial dysfunction. The causes, however, remain unclear.

Nitric oxide (NO) is a key player in the down regulation of platelet aggregation and in the regulation of blood vessel tonicity $[7,12,26]$. Measuring the NO concentrations revealed a considerable decrease $(72.5 \pm 25.6 \%)$ of NO-release from the HUAEC $24 \mathrm{~h}$ after stent impression in comparison to the NOrelease directly prior to stent impression. A decrease in NO-release of $72.5 \pm 25.6 \%$ from the baseline value was most remarkable. A similar argumentation as in the case of prostacyclin allows to conclude that the decrease in NO-release could not be expected regarding the small numbers of destroyed HUAEC and including a certain reduction in function of proliferating HUAEC. Future examinations will reveal and quantify the influence of stent impression on the inducible NO-release. Answering this question could be very important to elucidate the pathophysiology of early restenosis following PTCA with stent implantation.

The vitality of HUAEC in culture exposed to stents did not differ from control cultures. This could be concluded from the LDH measurements; $24 \mathrm{~h}$ after stent impression the LDH values, which are indicative of cell damage, showed the base line level.

Endothelial cells are actively involved in vascular repair and remodelling which occurs in certain cardiovascular diseases or after vessel wall injury. In this process involved are migration and proliferation of EC-precursors induced by a large number of compounds. In the supernatant of endothelial cells 1054 proteins were identified; of these, 239 proteins were of cytosolic and nuclear origin and were probably shed into the culture media due to cell death [3]. Therefore a lot of compounds - like different growth relevant factors [23] - influencing migration and proliferation of HUAEC can be expected in the direct vicinity of the stent wound where the concentration of such factors might be higher than in more remote regions.

BrdU incorporation as indicator of endothelial cell proliferation was carefully and completely examined around the stent impression - induced wound areas to unveil the influence of stent impression on local proliferation rates, and if re-occlusion phenomena (instent-restenoses), showing in vivo a certain asymmetry, already appear in static vascular models [2]. Such restenoses are often observed at the stent 
entry/exit ("candy wrapper" phenomenon [1]). The current discussion is attributing such restenoses to phenomena of the blood stream influenced by the stent geometry or the topography of the stent surface in the vascular wall $[22,29]$. However, there are different types of asymmetry. One type is evident regarding the proliferation rates near to the impression wound and remote from it. Adjacent to the impression wounds the highest proliferation rates were found. With increasing distance to the impression wound, smaller and smaller proliferation rates occurred. One reason for this is probably an increased release of growth enhancing factors by perishing HUAEC [23] from the wound area [28]. That allows to argue that HUAEC near to the border of wounded areas see more of the growth relevant factors and can be expected to show increased rates of proliferation, decreasing with dilution and consumption of these factors with increasing distance to the wound border. Contributing to this is most probably the lack of cell-cell-contacts of the HUAEC next to the wound area [21].

There was another significant difference and asymmetry in the view fields, which were farthest remote from the wound border: The numbers of proliferating HUAEC at the entry-/exit-sides were clearly greater than at the long sides of the stents.

The reason for this phenomenon in static cell cultures could be the plane omni- directional diffusion of the growth enhancing factors. These are released from perishing HUAEC and diffuse more or less radially from their sources. This could explain the almost symmetrical decrease in numbers of proliferating HUAEC at the long sides of the stents with increasing distance to the impression wound and almost parallel to the long stent sides. The diffusion field of the growth enhancing factors is probably more complex at the entrance-/exit- sides of the impression-induced wound. Radially diffusing growth enhancing factors from HUAEC of the long stent sides but near to the entrance-/exit sides will diffuse "around the corner" and into the cell covered areas orthogonal to the entrance-/exit-sides of the stents. In some distance from the stent-cell-border these growth enhancing factors coming from damaged HUAEC at the long sides of the stents could add to growth enhancing factors coming from damaged HUAEC at the entrance-/exit-sides of stents. This might explain the increase in numbers of proliferating HUAEC at the entrance-/exit-sides of stent impression-induced wounds some distance away from the wound border.

In conclusion it is important to note that the impression of stents in a vascular-wall-model consisting of human arterial endothelial cells on extracellular matrix (ECM) was followed by a significant decrease of prostacyclin- and NO-release indicative of an early impaired function of the endothelial cells. The massive reduction of the NO-release is thought to be a clear hint that similar mechanisms could be active in vivo contributing to the acute stent thrombosis $[13,19]$. However, HUAEC neighbouring the wound area remained vital and proliferation competent and were quickly regenerated. $24 \mathrm{~h}$ after stent impression the LDH values were found again on the base line level.

It could be hypothesized that the organisation of a complete healing of the endothelial lesion-concluded from cell vitality and increased proliferation activity - has priority over regulation of vascular tonicity and down regulation of platelet aggregation - concluded from the decrease in prostacyclin - and NO-release.

\section{References}

[1] Angiolillo DJ, Sabatá M, Alfonso F, Macaya C, "Candy wrapper” effect after drug-eluting stent implantation: Déjà vu or stumbling over the same stone again? Catheter Cardiovasc Interv 2004;61:387-91.

[2] Bach R, Die Auswahl und Implantation koronarer Stents. In: Trends in der invasiven Kardiologie. Akademische Verlagsgesellschaft Berlin 1997;1-7.

[3] Brown KJ, Seol H, Pillai DK, Sankoorikal B-J, Formolo CA, Mac J, Edwards NJ, Rose MC, Hathout Y. The human secretome atlas initiative: Implications in health and disease conditions. Biochimica et Biophysica Acta 2013;1834:2454-61. 
[4] Brust M, Schaefer C, Doerr R, Pan L, Garcia M, Arratia PE, Wagner C. Rheology of Human Blood Plasma: Viscoelastic Versus Newtonian Behavior. Phys Rev Lett 110:078305.

[5] Clague JR, Arvinder S, Kurbaan MB, et al. The new ACS Multi Link coronary stent: A single centre experience in 103 consecutive patients with and without oral anticoagulation. J Intervent Cardiol 1997;10:183.

[6] Dörler J, Frick M, Hilber M, Breitfuss H, Abdel-Hadi MN, Pachinger O, Liepsch D, Schwarzacher SP. Coronary stents cause high velocity fluctuation with a flow acceleration and flow reduction in jailed branches: An in vitro study using laser-Doppler anemometry. Biorheology 2012;49:329-40.

[7] Forconi S, Gori T. Endothelium and hemorheology. Clin Hemorheol Microcirc 2013;53:3-10.

[8] Franke RP, Gräfe M, Schnittler HJ, Seiffge D, Mittermayer C, Drenckhahn D. Induction of human vascular endothelial stress fibres by fluid shear stress. Nature 1984;307/5952:648-9.

[9] Franke RP, Bach R, Fuhrmann R, Jung F. Interaktionen zwischen Endothel und Koronarstent in einem Modell humaner Gefäße. In: Aktuelles aus der Klinischen Mikrozirkulation und Hämorheologie 1998. Jung, F.; Sternitzky, R.; Landgraf, H (Hrsg). AKA Verlag, Berlin 1999;250-7.

[10] Franke RP, Fuhrmann R, Mrowietz C, Rickert D, Hiebl B, Jung F. Reduced diagnostic value of lactate dehydrogenase (LDH) in the presence of radiographic contrast media. Clin Hemorheol Microcirc 2010;45:123-30.

[11] Galdal KS, Lyberg T, Evensen SA, Nilson E, Prydz H. Thrombin induces thromboplastin synthesis in cultured vascular endothelial cells. Thromb Haemost 1985;54:373-6.

[12] Gori T, Damaske A, Muxel S, Radmacher MC, Fasola F, Schaefer S, Fineschi M, Forconi S, Jung F, Münzel T, Parker JD. Endothelial function and hemorheological parameters modulate coronary blood flow in patients without significant coronary artery disease. Clin Hemorheol Microcirc 2012;52:255-66.

[13] Gori T, Münzel T. Endothelial dysfunction after stenting and scaffolding of coronary arteries. Clin Hemorheol Microcirc 2014;58:175-81.

[14] Gratzner HG, Leif RC, Ingram DJ, Castro A. The use of antibody specific for bromodeoxyuridine for the immunofluorescent determination of DNA replication in single cells and chromosomes. Exp Cell Res 1975;95:88.

[15] Jaffe EA, Nachman RL, Becker CG. Culture of human endothelial cells derived from umbilical cord veins. J ClinInvest 1972;51:46a.

[16] Jaffe EA, Nachman RL, Becker CG, Minick CR. Culture of human endothelial cells derived from umbilical cord veins. J Clin Invest 1973;52:2745-68.

[17] Jung F, Bach R, Franke RP. Topographische und Elementanalyse endovaskulärer Koronarstents. Minimal Invasive Medizin 1997;8:20-8.

[18] Korzwniewski C, Callewaert DM. An enzyme-release assay for natural cytocicity. J Immunol Methods 1983;64:313-20.

[19] Kranjec I, Dzananovic DZ. Acute ischemic events are frequent after primary coronary stenting. J Cardiovasc Med (Hagerstown) 2015;16:178-88.

[20] Krüger A, Braune S, Kratz K, Lendlein A, Jung F. The influence of poly(n-butyl acrylate) networks on viability and function of smooth muscle cells and vascular fibroblasts. Clin Hemorheol Microcirc 2012;52:283-94.

[21] Lampugnani MG, Zanetti A, Corada M, Takahashi T, Balconi G, Breviario F, Orsenigo F, Cattelino A, Kemler R, Daniel TO, Dejana E. The Journal of cell biology 2003;161:793.

[22] Lutter C, Nothhaft M, Rzany A, Garlichs CD, Cicha I. Effect of specific surface microstructures on substrate endothelialisation and thrombogenicity: Importance for stent design. Clin Hemorheol Microcirc 2015;59:219-33.

[23] McNeil PL, Muthukrishnan L, Warder E, D`Amore PA. Growth factors are released by mechanically wounded endothelial cells. The Journal of Cell Biology 1989;109:811-22.

[24] Maxey KM, Maddipati KR, Birkmeier J. Interference in Enzyme immuno-assay. J Clin Immunoassay 1992;15:116-20.

[25] Moncada S, Gryglewski R, Bunting S, Vane JR. An enzyme isolated from arteries trnasforms prostaglandin endoperoxides to an unstable substance that inhibits platelet aggregation. Nature 1976;263:663-5.

[26] Palmer RMJ, Ferrige AG, Moncada S. Nitric oxide accounts for the biollogical activity of endothelium derived relaxing factor. Nature 1987;327:524.

[27] Palmer RMJ, Ashton DS, Moncada S. Vascular endothelial cells synthesize nitric oxide from L-arginin. Nature 1988;333:664-6.

[28] Nelson CM, Chen CS. Journal of Cell Science 2003;116:3571.

[29] Poon EK, Barlis P, Moore S, Pan WH, Liu Y, Ye Y, Xue Y, Zhu SJ, Ooi AS. Numerical investigations of the haemodynamic changes associated with stent malapposition in an idealised coronary artery. J Biomech 2014;47:2843-51.

[30] Priestley KA, Clague JA, Buller NP, et al. First clinical experience with a new flexible low profile metallic stent and delivery system. Eur Heart J 1996;17:438-44. 
[31] Stern DM, Nawroth PP. Modulation of endothellal cell hemostatic properties by tumor necrosis factor. J Exp Med 1986;163:740-5.

[32] Ullm S, Krüger A, Tondera C, Gebauer TP, Neffe AT, Lendlein A, Jung F, Pietzsch J. Biocompatibility and inflammatory response in vitro and in vivo to gelatin-based biomaterials with tailorable elastic properties. Biomaterials 2014;35:9755-66.

[33] Weksler BB, Marcus AJ, Jaffe ES. Synthesis of prostaglandin I2 (prostazyklin) by cultured human endothelial cells. Proc Natl Acad Sci USA 1977;74:3922-6.

[34] Wong P, Wong CM, Chang CH, et al. Early clinical experience with the Multi Link coronary stent. Cathet Cardiovasc Diagn 1996;39:413.

[35] Zwaginga JJ, Sixma Jan J, Philip G. de Groot. Activation of endothelial cells induces platelet thrombus formation on their matrix studies of new in vitro thrombosis model with low molecular weight heparin as anticoagulant. Arteriosclerosis 1990;10:49-61. 